\title{
Unequal Decrease in Bone Density of Lumbar Spine and Ultradistal Radius in Colles' and Vertebral Fracture Syndromes
}

Richard Eastell, Heinz W. Wahner, W. Michael O'Fallon, Peter C. Amadio, L. Joseph Melton III, and B. Lawrence Riggs

Endocrine Research Unit, Section of Diagnostic Nuclear Medicine, Department of Health Sciences Research, and

Department of Orthopedics, Mayo Clinic and Mayo Foundation, Rochester, Minnesota 55905

\begin{abstract}
We measured bone mineral density (BMD) at the lumbar spine (LS-BMD) and ultradistal radius (UDR-BMD) in 42 postmenopausal normal women and in 108 postmenopausal osteoporotic women (55 with vertebral fracture, 34 with Colles' fracture, and 19 with both fractures). By receiver operating characteristic analysis, LS-BMD was better than UDR-BMD $(P<0.01)$ as an indicator of vertebral fracture; the converse was true for Colles' fracture $(P<\mathbf{0 . 0 1})$. Although UDR-BMD and LS-BMD were lower in each of the three fracture groups than in controls $(P<0.01)$, the pattern of bone loss differed $(P$ $<0.001$, analysis of variance): with vertebral fracture, LSBMD decreased relatively more than UDR-BMD; with Colles' fracture, UDR-BMD decreased relatively more than LSBMD; and with both fractures, decreases in LS-BMD and UDR-BMD were similar. We conclude that both types of fracture are caused by excessive bone loss but the difference in bone loss at the two sites is a major factor in determining which will fracture.
\end{abstract}

\section{Introduction}

Involutional osteoporosis has been divided into two types on the basis of differences in bone density, in the age- and sex-specific incidence pattern of the associated fractures, and in mechanisms of bone loss $(1,2)$. In type I osteoporosis, there is disproportionate and accelerated loss of trabecular bone, and fractures characteristically occur at skeletal sites containing large amounts of trabecular bone: the vertebrae and distal forearm (Colles' fracture). In type II osteoporosis, there is a more gradual thinning of both trabecular and cortical bone, leading to fractures of the hip, pelvis, and proximal humerus and to multiple wedge fractures of the vertebrae ("dowager's hump"). Type I osteoporosis mainly affects women within $25 \mathrm{yr}$ of menopause and is believed to result from factors related to estrogen deficiency. Type II osteoporosis affects men and women older than $75 \mathrm{yr}$ and is believed to result from factors related to aging (1).

Bone loss from the vertebrae in women with type I osteoporosis and vertebral fracture has been extensively studied (1). Much less is known about the extent of bone loss from the

Address reprint requests to Dr. Riggs, Endocrine Research Unit, Mayo Clinic, 200 First Street SW, Rochester, MN 55905.

Received for publication 16 February 1988 and in revised form 6 July 1988.

J. Clin. Invest.

(c) The American Society for Clinical Investigation, Inc.

0021-9738/89/01/0168/07 \$2.00

Volume 83, January 1989, 168-174 ultradistal radius (UDR), ${ }^{1}$ i.e., the distal $3 \mathrm{~cm}$ of the radius, in women with type I osteoporosis and Colles' fracture or the relationship of loss at this site to loss in the vertebrae. Most densitometric measurements have been made proximal to the usual site of Colles' fracture, in a region containing 5-50\% trabecular bone (3-9).

In the present study, we measured bone mineral density (BMD) of the lumbar spine (LS-BMD) and UDR (UDR$\mathrm{BMD}$ ) in women with type I osteoporosis in order to answer two questions. The first question was: is measuring UDRBMD as effective as measuring LS-BMD for detecting bone loss from the vertebrae in women with vertebral fracture? If trabecular bone loss were uniform throughout the skeleton and if cortical bone density were unchanged, then similar results would be obtained at measurement sites with similar proportions of trabecular bone. If measurement of UDRBMD provided a good estimate of LS-BMD, this would have practical advantages. The radius is considerably easier to measure than the spine and is not beset with artifacts such as spinal deformities (bone spurs, vertebral fracture) and aortic calcification that may confound the measurement of LS-BMD in older women. We addressed this question by performing receiver operating characteristic (ROC) analysis.

The second question was: if all women with type I osteoporosis undergo a phase of accelerated trabecular bone loss (1), why do some of them have Colles' fracture and others have vertebral fracture? We tested the hypothesis that unequal bone loss predisposes some osteoporotic women to vertebral fracture and others to Colles' fracture. The null hypothesis is that the degree of bone loss is similar at sites of predominantly trabecular bone, and that the type of fracture is determined only by external events such as trauma to one site or the other.

Bone loss from the lumbar vertebrae can be measured by dual-photon absorptiometry. However, in order to measure the relevant site in the UDR, it was necessary to develop a method for measuring BMD at the distal $3 \mathrm{~cm}$ of the radius. This site is composed of predominantly trabecular bone (10) and is where Colles' fracture occurs. The method uses singlephoton absorptiometry and computer-assisted image processing for accurate repositioning and selection of the region of interest.

\section{Methods}

\section{Experimental subjects}

Normal women. We studied 42 healthy postmenopausal women (Table I), aged 50-75 yr, who were 1-31 yr postmenopausal. None had had menopause before the age of $40 \mathrm{yr}$, had any disease or was taking

1. Abbreviations used in this paper: LS-BMD, lumbar spine bone mineral density; UDR-BMD, ultradistal radius bone mineral density; ROC, receiver operating characteristic. 
any medication known to affect bone density, or had a history of pain or stiffness of the wrist. No subject had a vertebral fracture evident on anteroposterior or lateral radiographs of the lumbar and thoracic spine.

Fracture groups. The patients with type I osteoporosis were divided into three groups according to the type of fractures they had.

The first group (55 women, aged 54-75 yr) had only vertebral fracture. All had three or more mild wedge fractures (anterior vertebral height $75-85 \%$ of posterior height) or one or more severe wedge fractures (anterior height $<75 \%$ of posterior height) or both. All fractures had occurred after minimal or no trauma. Factors that might have resulted in decreased bone density included a history of thyrotoxicosis in one, chronic obstructive airway disease in one, and surgically induced premature menopause in seven. None had had previous treatment with fluoride, but 28 were being treated with calcium supplements $(0.5-2 \mathrm{~g}$ of elemental calcium daily) and 10 had previously used or currently were using estrogen.

The second group (34 women, aged 53-75 yr) had only Colles' fracture. The fractures had occurred 1-28 mo (mean, $11.7 \mathrm{mo}$ ) before measurement; they affected the dominant forearm in 17 and the nondominant forearm in 17. Factors that might have contributed to decreased bone density included a history of thyrotoxicosis in two, chronic obstructive airway disease in two, and surgically induced premature menopause in three. None were being treated for bone loss.

The third group (19 women, aged 59-75 yr) had both Colles' and vertebral fractures. The Colles' fracture had occurred 1-31 yr (mean, $15 \mathrm{yr}$ ) before measurement and affected the dominant forearm in 8 and the nondominant forearm in 11 . The criteria for vertebral fracture were the same as for the first group. Factors that may have resulted in decreased bone density included a history of thyrotoxicosis in one and surgically induced premature menopause in one. None had had previous treatment with fluoride or estrogen, but 10 were being treated with calcium supplements (0.5-2 $\mathrm{g}$ of elemental calcium daily).

\section{Bone densitometry}

UDR-BMD was measured in the nondominant forearm (or in the uninjured forearm in the Colles' fracture group) by single-photon absorptiometry with computer-assisted image processing. The forearm was positioned in the scanning apparatus, and the wrist was surrounded by a water bag to ensure constant thickness over the entire scanning path. Scanning of the distal $3 \mathrm{~cm}$ of the radius began at the radial styloid process (identified by palpation) and moved proximally in 2-mm steps (that is, 15 scan lines). The image (Fig. $1 \mathrm{~A}$ ) was displayed on a microcomputer (IBM-PC) to confirm correct positioning of the wrist and then was stored on a floppy disc for subsequent processing. The radiation source was ${ }^{125} \mathrm{I}$ (photopeak, $27 \mathrm{keV}$ ) and the collimator was $2 \mathrm{~mm} \times 2 \mathrm{~mm}$.

The image display program allowed selection of regions of interest and detection of bone edges. The area of interest was $1 \mathrm{~cm}$ long (Fig. 1 $B$ ), and the distal end of this area was $4 \mathrm{~mm}$ proximal to the medial edge of the distal articular surface of the radius (which was always the line of peak bone density). We chose this particular site because it is the site through which Colles' fracture occurs. We determined that Colles'

Table I. Characteristics of Patient Groups

\begin{tabular}{lcccc}
\hline & & \multicolumn{3}{c}{ Osteoporotic women } \\
\cline { 3 - 5 } \multicolumn{1}{c}{ Variable } & Normal women & $\begin{array}{l}\text { Vertebral } \\
\text { fracture }\end{array}$ & $\begin{array}{c}\text { Colles' } \\
\text { fracture }\end{array}$ & $\begin{array}{c}\text { Both } \\
\text { fractures }\end{array}$ \\
\hline Number & 42 & 55 & 34 & 19 \\
Age (yr) & $62 \pm 8$ & $67 \pm 5$ & $65 \pm 6$ & $68 \pm 5$ \\
Postmenopause (yr) & $12 \pm 8$ & $19 \pm 7$ & $16 \pm 7$ & $21 \pm 9$ \\
Weight (kg) & $68 \pm 13$ & $63 \pm 11$ & $70 \pm 13$ & $65 \pm 10$
\end{tabular}

Values given as mean $\pm \mathrm{SD}$. fracture occurs at a mean distance of $22 \mathrm{~mm}( \pm 4 \mathrm{~mm}, \mathrm{SD})$ proximal to the tip of the radial styloid, based on measurements made on 20 radiographs of Colles' fracture with minimal displacement.

Precision of UDR-BMD, determined by duplicate measurements on 20 premenopausal healthy subjects, was $1.7 \% .^{2}$ The value for the dominant radius was $3 \%$ higher, on average, than that for the nondominant radius as determined by measurements made on another 20 healthy premenopausal women. Thus, in women with Colles' fracture of the nondominant forearm, the dominant forearm was measured and a $3 \%$ correction was made.

LS-BMD was determined by dual-photon absorptiometry of vertebrae L-2 to L-4 with the scanning apparatus previously described (11) and ${ }^{153} \mathrm{Gd}$ as the source (photopeaks, 44 and $100 \mathrm{keV}$ ). Fractured vertebrae were not measured. The precision was $2.2 \%$.

\section{Anatomic studies of the radius}

Radii removed from four cadavers were studied to determine the accuracy of the measurement and the proportion of trabecular bone at the site of measurement. To determine BMD of the bone specimens, after removal of surrounding tissue they were air-dried, defatted, and embedded in methyl methacrylate. Three to five pieces $2-7 \mathrm{~mm}$ wide were cut with a jeweler's saw (Isomet, Buehler Ltd., Lake Bluff, IL), and $200-\mu \mathrm{m}$ sections from the proximal end of the pieces were ground to $100-\mu \mathrm{m}$ sections before microradiography; the proportion of trabecular bone was estimated by point counting (Table II; Fig. 2). The remaining pieces of bone were partially ashed $\left(500^{\circ} \mathrm{C}\right.$ for $\left.24 \mathrm{~h}\right)$; then, trabecular bone was separated from cortical bone by dissection and ashed in a muffle furnace at $600^{\circ} \mathrm{C}$ for $24 \mathrm{~h}$. The amount of each type of bone was determined by weighing.

The results of the accuracy analysis are shown in Fig. 3. The regression line does not intersect the $y$-axis because the cadaver radii were scanned with the marrow fat in situ: this method allows correction for the effect of marrow fat in vivo. The regression line for defatted bone is shown for comparison (12). The accuracy of the technique (the standard error of the estimate of the regression $\times 100 /$ mean) was $8 \%$.

\section{Statistical analysis}

The ability of the two BMD measurements to discriminate between the normal subjects and women with vertebral fracture or women with Colles' fracture was evaluated by applying the ROC curve approach $(13,14)$. For each of the two BMD measurements and for each fracture group, all possible cut-off points were defined and the proportion of healthy subjects above (the specificity) and the proportion of osteoporotic subjects below (the sensitivity) each point were calculated. This yields an ROC curve that displays the relationship between sensitivity and specificity for each BMD measurement as a discriminator between the normal and fracture groups. The area between two ROC curves contrasts the ability of two BMD measures to discriminate. The areas were estimated and tested (null hypothesis is that area equals 0 ) by a technique developed by Wieand et al. ${ }^{3}$

The results of LS-BMD and UDR-BMD were reported in grams per square centimeter and in the form of $Z$ scores. $Z$ scores were used for two reasons. First, the BMD result was adjusted for factors that differ between individuals, such as age and weight. Secondly, this approach converts the deviation from normal of BMD values at each of the two scanning sites into SD units and thus allows estimation of the relative deficit in BMD in each fracture group.

$Z$ scores were calculated by a two-step procedure. First, the effects of age, (age $)^{2},(\text { age })^{3}$, body weight, height, and years postmenopause on $\mathrm{BMD}$ in the 42 normal women were assessed by multiple regression analysis. Both LS-BMD and UDR-BMD correlated most closely with

2. Precision $=(S D$ of differences between paired $B M D$ measurements)/(mean BMD) $\times 100$.

3. Wieand, H. S., K. James, B. James, and M. H. Gail. 1987. Nonparametric procedures for comparing diagnostic tests with paired or unpaired data. Unpublished data. 

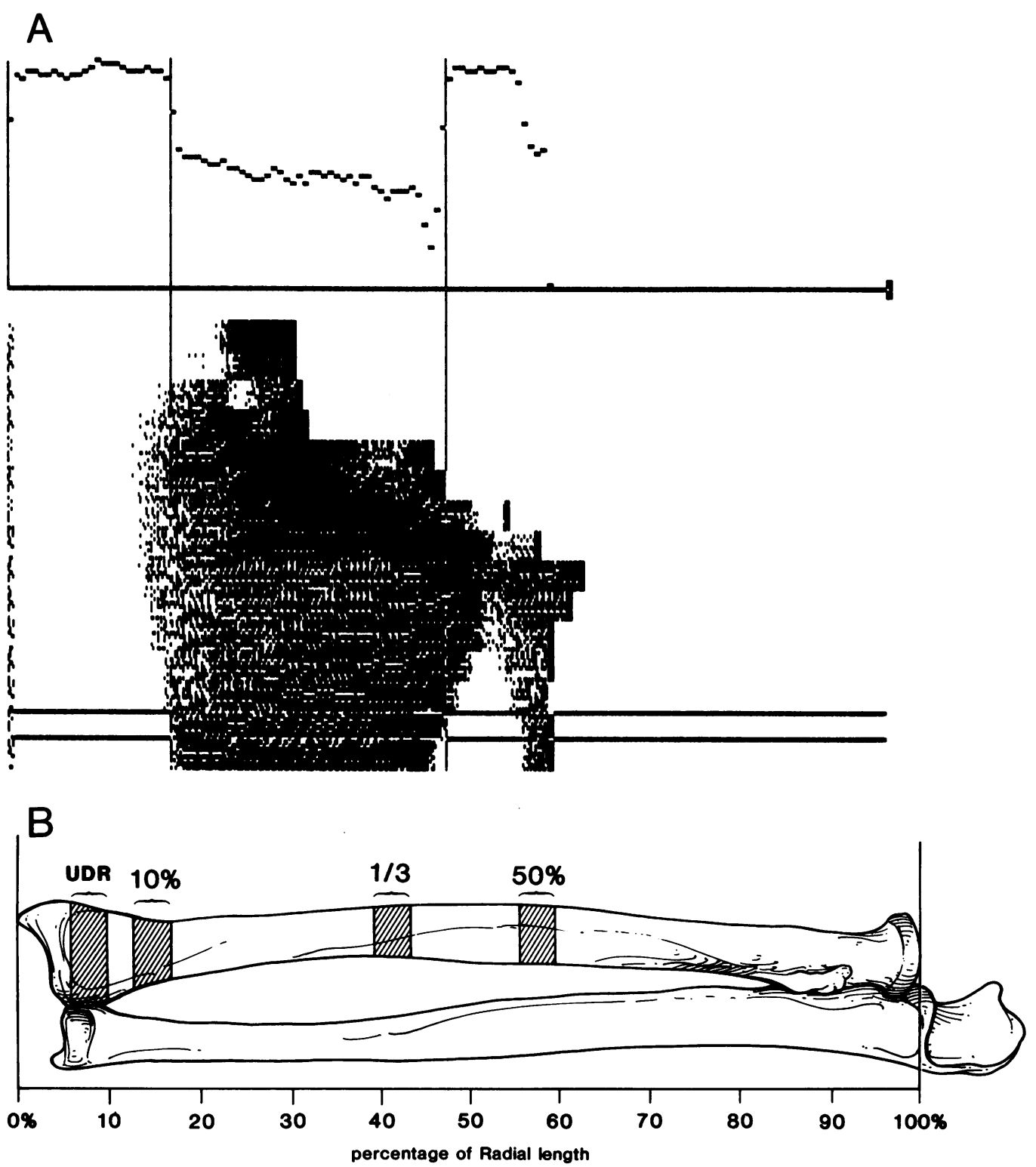

Figure 1. (A) Computer-assisted image of distal $3 \mathrm{~cm}$ of radius. Use of horizontal and vertical cursors allows selection of region of interest. Upper panel displays BMD profile ( $y$-axis is logarithm of attenuation; $x$ axis is scan distance) selected by horizontal cursors. (B) Tracing of radiograph of forearm bones excised postmortem from 94-yr-old man to show site of measurement of UDR-BMD. In previous studies (11) BMD was measured at the midradius (50\%), one-third site, and distal site $(10 \%)$; these proportions relate to distance along the ulna, not along the radius. age and body weight: $R^{2}=0.36(P<0.001)$ and $0.29(P<0.01)$, respectively. Secondly, the regression equations were used to predict BMD based on an individual's age and body weight. The $Z$ score for BMD at either site was then calculated as $Z$ score $=$ (observed BMD

\section{Table II. Trabecular Bone Content in Radii from Four Cadavers}

\begin{tabular}{ccccc}
\hline \multicolumn{2}{c}{ Cadaver } & & & \multicolumn{2}{c}{ Trabecular bone } \\
\cline { 4 - 5 } Age at death & Sex & & By weighing & By point counting \\
\hline$y r$ & & & \multicolumn{2}{c}{$\%$} \\
33 & M & 53 & 72 \\
52 & M & 74 & 74 \\
74 & F & 60 & $*$ \\
94 & M & 56 & 71 \\
Mean & & 61 & \\
& & &
\end{tabular}

* Samples fractured during preparation for microradiography.
- predicted BMD)/( $\left.s_{y \cdot x}\right)$ in which $s_{y \cdot x}$ is the standard deviation of BMD values about the regression line. Thus, by definition, the mean $Z$ score in normal subjects would be 0 , and $95 \%$ of normal subjects would have $Z$ scores between -2 and +2 .

The $Z$ score calculations were used to answer two questions. First, did BMD in the fracture groups differ from BMD in the normal group? Under the null hypothesis that the fracture groups are the same as the normal group, the mean $Z$ score should not differ significantly from 0 ; this hypothesis was tested by using one-sample $t$ tests. Secondly, was there relatively greater loss of LS-BMD in the vertebral fracture group and relatively greater loss of UDR-BMD in the Colles' fracture group? This hypothesis was tested by calculating the mean difference between LS-BMD $Z$ score and UDR-BMD $Z$ score among each of the fracture groups. Thus, if there was relatively greater bone loss at LS than at UDR, the difference LS-BMD $Z$ score minus UDR-BMD $Z$ score would be negative; conversely, if there was relatively greater bone loss at UDR than LS, the difference LS-BMD $Z$ score minus UDR-BMD $Z$ score would be positive. This derived value was used as a basis for statistical comparison of the relative degree of bone loss at the two scanning sites among groups. After testing for overall differences among the groups by one-way analysis of variance, we compared differences between groups by two-sample $t$ tests. 


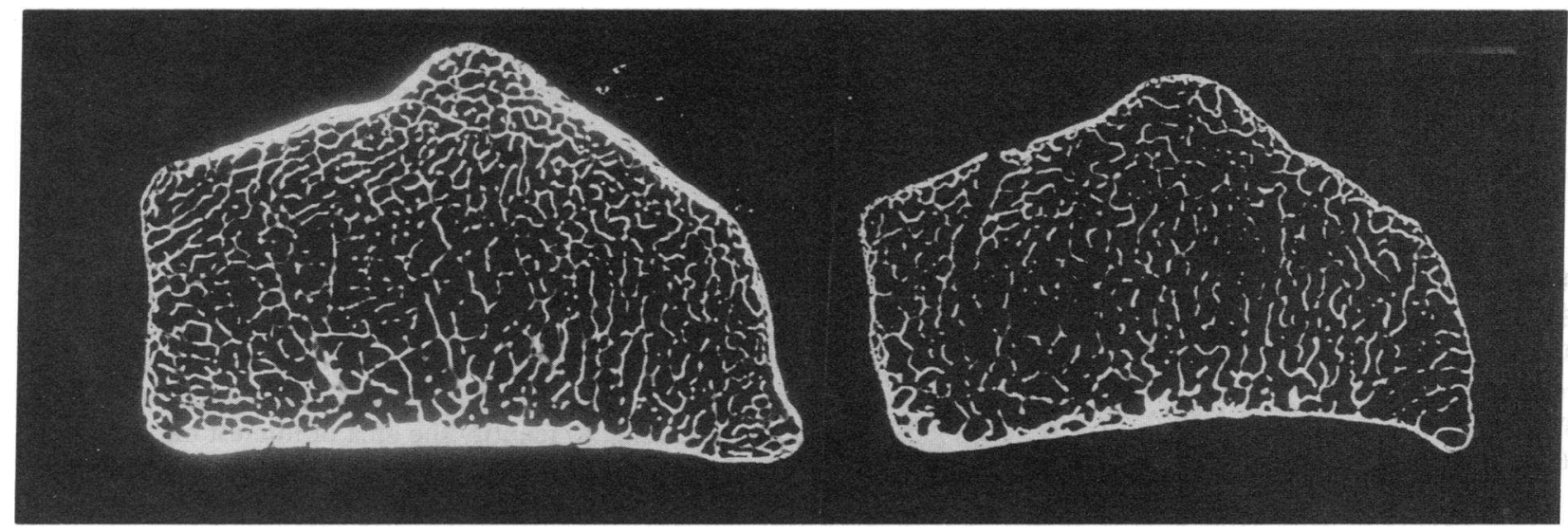

Figure 2. Microradiograph of $100-\mu \mathrm{m}$ sections from the middle of the region of interest in cadaver radius specimens. (Left) Section from 33-year-old man; (right) from 94-year-old man.

\section{Results}

Trabecular bone content of UDR. At the UDR site the mean percentage of trabecular bone was $71 \%$ by volume and $61 \%$ by weight (Table II).

Discrimination of fracture groups. The ROC curves for LSBMD and UDR-BMD in women with vertebral fracture are shown in Fig. $4 A$. For this analysis, the curve that is nearest to the top left corner (this corner corresponds to $100 \%$ sensitivity and $100 \%$ specificity) represents the best test. Thus, LS-BMD discriminates osteoporotic women with vertebral fracture from age-matched normal women better than UDR-BMD does $(P<0.001)$. The areas under the curves for LS-BMD and UDR-BMD were $91 \%$ and $78 \%$, respectively. The ROC curves for LS-BMD and UDR-BMD for women with Colles' fracture are shown in Fig. $4 B$. Here UDR-BMD discriminates better between women with and those without Colles' fracture than does LS-BMD $(P<0.01)$. The areas under the curves for UDR-BMD and LS-BMD were $73 \%$ and $61 \%$, respectively. The sensitivity and specificity of the two measurements in the

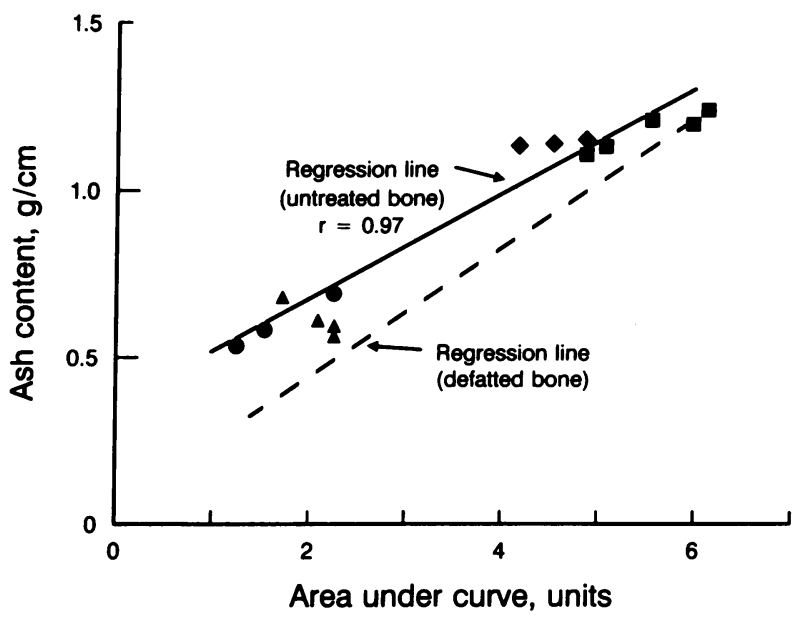

Figure 3. Relationship between estimated BMD (area under curve [see Fig. 1], in arbitrary units) and ash weight per unit length $(\mathrm{g} / \mathrm{cm})$ in four cadaver radius specimens. Regression line intersects $y$-axis above the origin. Equation used to calibrate the densitometer was: ash content $=0.161 \times($ bone density $)+0.318$. vertebral fracture and Colles' fracture groups are given numerically in Table III.

Unequal bone loss at sites of fracture. The LS-BMD and UDR-BMD values are given in Table IV. The percent decreases of mean LS-BMD from normal women were $25 \%, 7 \%$, and $25 \%$, respectively, in the groups with vertebral fracture, Colles' fracture, or both fractures. The respective percent decreases of mean UDR-BMD from normal women were $15 \%$, $12 \%$, and $20 \%$. All of these decreases were statistically signifi-

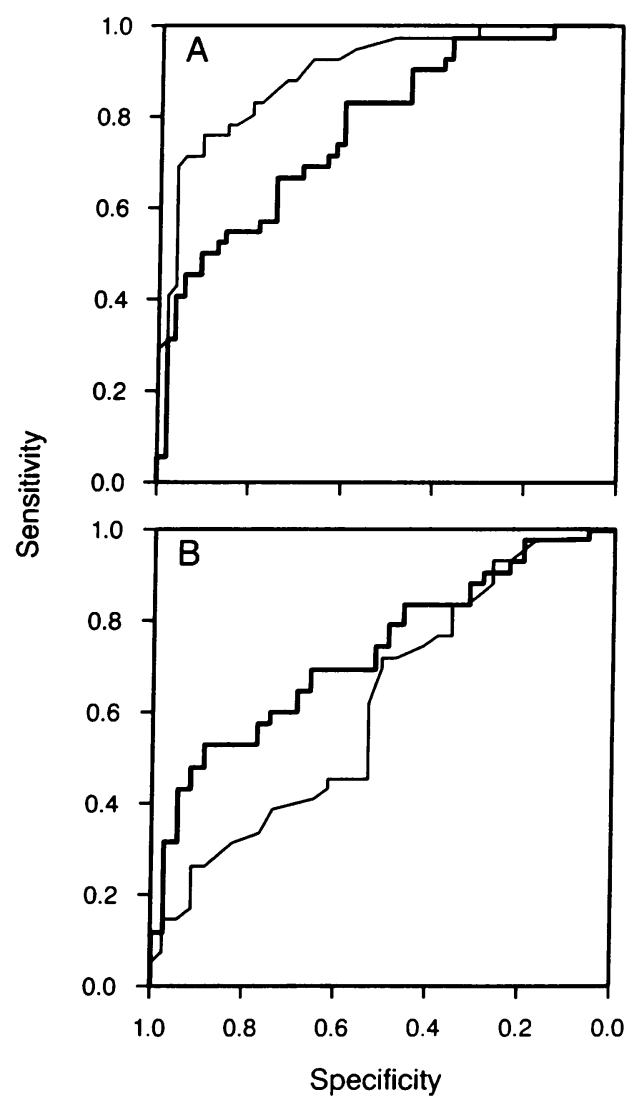

Figure 4. ROC curves showing sensitivity and specificity of LS-BMD (thin line) and UDR-BMD (thick line). ( $A$ ) In women with vertebral fractures. $(B)$ In women with Colles' fractures only. 
Table III. Sensitivity and Specificity of UDR-BMD and LS$B M D$ as Tests for Vertebral Fracture and Colles' Fracture

\begin{tabular}{lccccc}
\hline & \multicolumn{2}{c}{ Sensitivity* } & & \multicolumn{2}{c}{ Specificity } \\
\cline { 2 - 3 } \cline { 5 - 6 } Measurement & At 90\% & At 50\% & & At 90\% & At 50\% \\
& & & $\%$ & & \\
For vertebral fracture & & & & & \\
UDR-BMD & 45 & 90 & & 50 & 83 \\
LS-BMD & 69 & 96 & 76 & 98 \\
For Colles' fracture & & & & \\
UDR-BMD & 28 & 88 & 47 & 74 \\
LS-BMD & 26 & 53 & 27 & 72 \\
& & & & &
\end{tabular}

* At specificities of $90 \%$ and $50 \%$.

At sensitivities of $90 \%$ and $50 \%$.

cant (Table IV). In the group with vertebral fracture there was a relatively greater decrease in LS-BMD, in the group with Colles' fracture there was a relatively greater decrease in UDR-BMD, and in the group with both fractures there were similar decreases in both LS-BMD and UDR-BMD.

The women with vertebral fracture had the most negative mean difference $(-0.76)$ between LS-BMD $Z$ score and UDRBMD $Z$ score, those with Colles' fracture had the most positive mean difference $(0.40)$, and those with both fractures had an intermediate mean difference $(-0.22)$. These differences among the three mean values were statistically significant $(P$ $<0.001$, analysis of variance). The women with vertebral fracture alone and those with both fractures had a relatively greater decrease at LS-BMD than at UDR-BMD compared with women with Colles' fracture $(P<0.001, P<0.02$, respectively). In both groups of women with vertebral fracture, the mean decreases in LS-BMD were similar (Table IV); however, those with both vertebral and Colles' fractures had a marginally greater reduction in UDR-BMD than did those with vertebral fracture alone $(P=0.06)$.

Individual values for these relative differences are shown in Fig. 5. For the group with vertebral fracture only, $80 \%$ of the points fall below the line of identity, indicating that bone loss was relatively greater at LS than at UDR. For the group with Colles' fracture, $68 \%$ of the points fall above the line of identity, indicating that bone loss was relatively greater at UDR than at LS. For the group with both fractures, 9 points fall above the line of identity and 10 points fall below, indicating similar relative decreases at LS and UDR.

\section{Discussion}

We encountered two technical problems in developing a reproducible and accurate technique for measurement at the UDR. First, repositioning was found to be of critical importance at this site because there were large changes in bone mineral content and in the proportion of trabecular bone over a short distance. We overcame this by utilizing computer-assisted image processing to identify the area of interest on the intensity-modulated bone mineral image. Some investigators have attempted to solve this problem by scanning at a fixed distance between the radius and ulna (for example, at $5 \mathrm{~mm} \mathrm{[15]} \mathrm{or} 8$ $\mathrm{mm}$ [16]). However, at these sites the bone is less than $50 \%$ trabecular and the proportion of trabecular bone is very variable. Others have performed a preliminary "scout scan" by computed tomography $(5,17,18)$.

The second problem was that the high proportion of trabecular bone (Fig. 2) is associated with a large amount of fat in the marrow space, and this produces a systematic error in results of scanning with a single-energy photon source. This problem was appreciated by Karjalainen (19) but has been ignored by subsequent workers $(13,14)$. We corrected for it by measuring UDR-BMD in cadaver radius specimens with marrow fat in situ and then defatting the bone and ashing the specimen. This regression of ash content on area under the curve was then used to estimate UDR-BMD. The presence of fat has a marked effect on UDR-BMD because attenuation of the photon beam is less through fat than through water; thus, failure to correct for fat within the marrow space results in an underestimate of UDR-BMD.

Most previous studies of bone loss from the distal radius in patients with Colles' fracture scanned at sites proximal to the fracture site $(3,4,6-9)$, where the bone is mainly cortical; at those sites bone mineral content was $5-7 \%$ below normal. The one exception was the study by Hesp et al. (5) in which UDRBMD was measured by a combined computed tomography/ single photon absorptiometry technique. It is notable that they reported a relatively large decrease $(12 \%)$ in UDR-BMD as compared with age- and sex-matched normal subjects. In the present study, we also found that the mean decrease in UDRBMD in Colles' fracture patients was $12 \%$.

Table IV. Bone Density Values in Normal Women and Women With Fractures

\begin{tabular}{|c|c|c|c|c|}
\hline \multirow[b]{2}{*}{ Variable } & \multirow[b]{2}{*}{ Normal women } & \multicolumn{3}{|c|}{ Osteoporotic women } \\
\hline & & Vertebral fracture & Colles' fracture & Both fractures \\
\hline \multicolumn{5}{|l|}{ Absolute bone mass } \\
\hline $\operatorname{LS}-B M D\left(\mathrm{~g} / \mathrm{cm}^{2}\right)$ & $1.04 \pm 0.02$ & $0.78 \pm 0.02$ & $0.97 \pm 0.03$ & $0.78 \pm 0.03$ \\
\hline LS-BMD (\% below normal) & & 25 & 7 & 25 \\
\hline UDR-BMD $\left(\mathrm{g} / \mathrm{cm}^{2}\right)$ & $0.41 \pm 0.01$ & $0.35 \pm 0.01$ & $0.36 \pm 0.01$ & $0.33 \pm 0.01$ \\
\hline UDR-BMD (\% below normal) & & 15 & 12 & 20 \\
\hline \multicolumn{5}{|l|}{$Z$-scores } \\
\hline LS-BMD (SD units) & - & $-1.61 \pm 0.13^{*}$ & $-0.60 \pm 0.18^{\ddagger}$ & $-1.63 \pm 0.20^{*}$ \\
\hline UDR-BMD (SD units) & - & $-0.85 \pm 0.13^{*}$ & $-0.97 \pm 0.13^{*}$ & $-1.40 \pm 0.22^{*}$ \\
\hline
\end{tabular}

Values given as mean $\pm \mathrm{SE}$.

* For difference from normal women, $P<0.001$. ${ }^{\ddagger}$ For difference from normal women, $P<0.01$. 

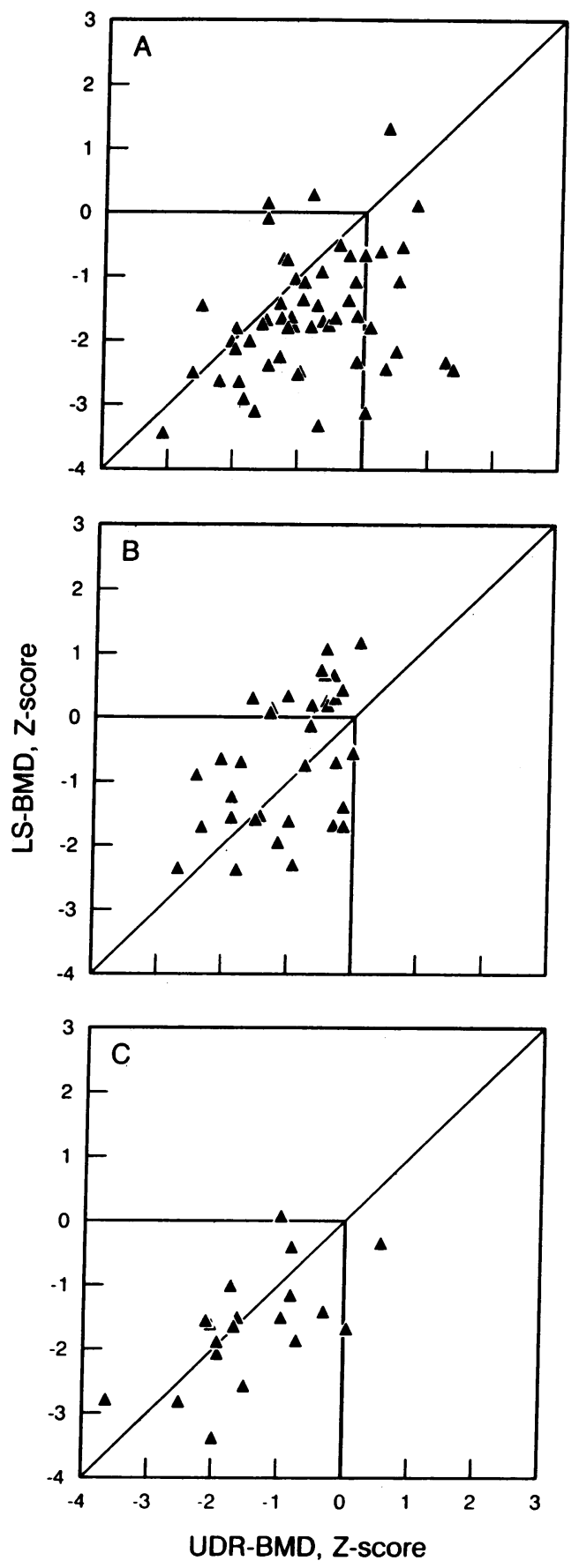

Figure 5. LS-BMD and UDR-BMD expressed as Z-scores, in individual women. Oblique line indicates line of identity. $(A)$ Women with vertebral fractures. $(B)$ Women with Colles' fractures. $(C)$ Women with both vertebral and Colles' fractures.

In our study, BMD was measured at sites composed mainly of trabecular bone. Our anatomic studies indicated that the UDR-BMD measurement site contained about $70 \%$ trabecular bone, and others $(10,20,21)$ obtained similar values. Most studies have found that vertebrae contain about $70 \%$ trabecular bone $(22,23)$. However, in a recent study of cadavers of eight elderly women (aged 60-86 years), Nottestad et al. (24) found the proportion of trabecular bone to be $42 \%$ in the body and $24 \%$ in the entire vertebrae. This low proportion of trabecular bone may have been related to age and to bone loss during the terminal illness.
Our findings provide answers to the two questions that we posed in the Introduction. In answer to the first question, UDR-BMD is not an appropriate substitute for LS-BMD in assessing the extent of bone loss from the vertebrae in women with vertebral fracture. ROC curve analysis showed that LSBMD measurements were much more sensitive and specific than UDR-BMD measurements for separating osteoporotic patients with vertebral fracture from age- and sex-matched normal controls, despite the similarity in content of trabecular bone at the two sites. Conversely, UDR-BMD measurements were more sensitive and specific than LS-BMD measurements for separating osteoporotic patients with Colles' fracture from normals. Thus, in order to predict fracture risk in a bone, the BMD of that bone should be measured-measurements made at other parts of the skeleton have less predictive value.

The results of LS-BMD measurements in women with vertebral fracture were similar to those in our earlier report (11); in that study, $45 \%$ of women with vertebral fracture had LSBMD values less than the 5th percentile of normals, compared with $49 \%$ in the present study ( $Z$ score $\leq 1.645$ ). In that study, $7 \%$ had distal radius BMC values less than the 5th percentile, compared with $25 \%$ with UDR-BMD values less than the 5 th percentile ( $Z$ score $\leq 1.645$ ). The higher proportion of trabecular bone at the UDR than at the distal radius may allow better separation of women with and without vertebral fracture.

These results do differ from those in two recent reports. Ott et al. (25) measured LS-BMD and BMC of the distal radius in postmenopausal women with and without vertebral fracture. Using ROC curve analysis, they found that the two measurement sites gave approximately equal sensitivity and specificity. Thus, for a sensitivity of $90 \%$ (i.e., the value we use as our "fracture threshold"), the specificity of LS-BMD in their study was $21 \%$, whereas in our study it was $76 \%$; the specificity of distal radius BMC in their study was $35 \%$, whereas for UDRBMD in our study it was $50 \%$. Thus, the major difference between our findings and those of Ott et al. (25) is better specificity of LS-BMD in our study. Nilas et al. (26) reported BMD results in 28 women with vertebral fracture. The mean LS-BMD $Z$ score was -0.43 (it was -1.61 in the present study), and the mean UDR-BMD $Z$ score was $-0.51(-0.85$ in the present study).

The results of Ott et al. (25) and Nilas et al. (26) may differ from our results for two reasons. We used stricter criteria for diagnosing osteoporosis, requiring the presence of at least three mild anteriorly wedged vertebrae; Nilas et al. (26) required only one such fracture and Ott et al. (25) required only two such fractures. Recently, the Danish group have further analyzed their data. By dividing their osteoporotic subjects into those with and without compression fractures, Podenphant et al. (27) now report a relatively greater loss of bone density at the lumbar spine in subjects with compression fractures. The precision of LS-BMD in the present study was 2.2\% (11); Nilas et al. (26) reported a precision of $6.2 \%$, and Ott et al. (25) did not report the precision of their method, but the intrapopulation standard deviation was greater than that reported by others (28).

With respect to the second question, differential bone loss at the site of fracture may explain, in part, why some women with type I osteoporosis have vertebral fracture and others have Colles' fracture. Why is there relatively greater bone loss at the LS in women with vertebral fracture and greater bone loss at the UDR in those with Colles' fracture, given that these sites are both composed mainly of trabecular bone? One expla- 
nation is that the differences were present at skeletal maturity ("peak bone mass") and that subsequent rates of bone loss were similar. An alternative explanation is that there were differences in the subsequent rates of bone loss at sites among individuals. The rates of bone loss may differ between the LS and the UDR because of the different forces to which these bones are subjected: bone loss from the spine may be decreased by weight-bearing exercise and obesity. Also, the rate of bone turnover in the LS may be greater than that in the UDR because the vertebrae contain both red cellular marrow (containing the precursors of osteoclasts and osteoblasts) and yellow fatty marrow whereas the UDR contains only the latter. The proportion of red to yellow marrow in the vertebrae may differ between individuals and could account for differences in rate of bone loss.

Osteoporotic women with only vertebral fracture had a much larger mean decrease in LS-BMD than did those with only Colles' fracture. However, the mean decrease in UDRBMD was only slightly larger in women with Colles' fracture than in those with vertebral fracture. Thus, the women with vertebral fracture are also at increased risk for Colles' fracture but have not yet sustained the necessary trauma such as falling on the outstretched hand. An alternative explanation is that women with Colles' fracture are more likely to fall than those with vertebral fracture. In this regard, Crilly et al. (7) reported that women with Colles' fracture had more postural instability than age-matched controls.

In summary, although patients with type I osteoporosis lose excessive amounts of bone from both the LS and the UDR, the amounts of bone lost at these two sites vary among individuals. The relative decreases at these two sites may contribute to the development of the different fracture syndromes. Whatever the underlying cause of the unequal bone loss in type I osteoporosis, it appears that site-specific measurements of BMD are the best way to study these fracture syndromes and the best way to estimate fracture risk prospectively.

\section{Acknowledgments}

We thank Joan M. Muhs for coordinating the patient studies; Darla J. Jech, Robert L. Carlson, and William L. Dunn for assisting with bone density measurements; and Cheryl K. Collins for preparing the manuscript.

This investigation was supported in part by research grant AR-27065 from the National Institutes of Health.

\section{References}

1. Riggs, B. L., and L. J. Melton III. 1986. Involutional osteoporosis. N. Engl. J. Med. 314:1676-1686.

2. Riggs, B. L., and L. J. Melton III. 1983. Evidence for two distinct syndromes of involutional osteoporosis. Am. J. Med. 75:899-901.

3. Nilsson, B. E., and N. E. Westlin. 1974. The bone mineral content in the forearm of women with Colles' fracture. Acta Orthop. Scand. 45:836-844.

4. Jensen, G. F., C. Christiansen, J. Boesen, V. Hegedüs, and I. Transbol. 1983. Relationship between bone mineral content and frequency of postmenopausal fractures. Acta Med. Scand. 213:61-63.

5. Hesp, R., L. Klenerman, and L. Page. 1984. Decreased radial bone mass in Colles' fracture. Acta Orthop. Scand. 55:573-575.

6. Härmä, M., and P. Karjalainen. 1986. Trabecular osteopenia in Colles' fracture. Acta Orthop. Scand. 57:38-40.

7. Crilly, R. G., L. Delaquerriere Richardson, J. H. Roth, A. A. Vandervoort, K. C. Hayes, and R. A. Mackenzie. 1987. Postural stability and Colles' fracture. Age Ageing. 16:133-138.

8. Nordin, B. E. C., R. G. Crilly, and D. A. Smith. 1984. Osteoporo- sis. In Metabolic Bone and Stone Disease. 2nd edition. B. E. C. Nordin, editor. Churchill Livingstone, Edinburgh. 1-70.

9. Krolner, B., E. Tondevold, B. Toft, B. Berthelsen, and S. P. Nielsen. 1982. Bone mass of the axial and the appendicular skeleton in women with Colles' fracture: its relation to physical activity. Clin. Physiol. (Oxf.). 2:147-157.

10. Schlenker, R. A., and W. W. VonSeggen. 1976. The distribution of cortical and trabecular bone mass along the lengths of the radius and ulna and the implications for in vivo bone mass measurements. Calcif. Tissue Res. 20:41-52.

11. Riggs, B. L., H. W. Wahner, W. L. Dunn, R. B. Mazess, K. P. Offord, and L. J. Melton III. 1981. Differential changes in bone mineral density of the appendicular and axial skeleton with aging: relationship to spinal osteoporosis. J. Clin. Invest. 67:328-335.

12. Wahner, H. W., B. L. Riggs, and J. W. Beabout. 1977. Diagnosis of osteoporosis: usefulness of photon absorptiometry at the radius. J. Nucl. Med. 18:432-437.

13. Hanley, J. A., and B. J. McNeil. 1982. The meaning and use of the area under a receiver operating characteristic (ROC) curve. Radiology. 143:29-36.

14. Hanley, J. A., and B. J. McNeil. 1983. A method of comparing the areas under receiver operating characteristic curves derived from the same cases. Radiology. 148:839-843.

15. Awbrey, B. J., P. C. Jacobson, S. A. Grubb, W. H. McCartney, L. M. Vincent, and R. V. Talmage. 1984. Bone density in women: a modified procedure for measurement of distal radial density. $J$. Orthop. Res. 2:314-321.

16. Nilas, L., J. Borg, A. Gotfredson, and C. Christiansen. 1985. Comparison of single- and dual-photon absorptiometry in postmenopausal bone mineral loss. J. Nucl. Med. 26:1257-1262.

17. Rüegsegger, P., M. Anliker, and M. Dambacher. 1981. Quantification of trabecular bone with low dose computed tomography. $J$. Comput. Assist. Tomogr. 5:384-390.

18. Hangartner, T. N., and T. R. Overton. 1982. Quantitative measurement of bone density using gamma-ray computed tomography. J. Comput. Assist. Tomogr. 6:1156-1162.

19. Karjalainen, P. 1973. A method for determination of the mineral content and mineral density of the distal radius using gamma ray attenuation. Ann. Clin. Res. 5:231-237.

20. Melsen, F., H. E. Nielsen, P. Christensen, and L. Mosekilde. 1978. Some relations between photon-absorptimetric and histomorphometric measurements of bone mass in the forearm. Am. J. Roentgenol. 131:541. (Abstr.)

21. Nilas, L., H. Norgaard, J. Podenphant, A. Gotfredsen, and C. Christiansen. 1987. Bone composition in the distal forearm. Scand. $J$. Clin. Lab. Invest. 47:41-46.

22. Johnson, L. C. 1964. Morphologic analysis in pathology: the kinetics of disease and general biology of bone. In Bone Biodynamics. H. M. Frost, editor. Little, Brown and Company, Boston. 550.

23. Pesch, H.-J., H.-P. Scharf, G. Lauer, and H. Siebold. 1980. Der altersabhängige Verbundbau der Lendenwirbelkörper: eine Strukturund Formanalyse. Virchows Arch. A Pathol. Anat. Histopathol. 386:21-41.

24. Nottestad, S. Y., J. J. Baumel, D. B. Kimmel, R. R. Recker, and R. P. Heaney. 1987. The proportion of trabecular bone in human vertebrae. J. Bone Miner. Res. 2:221-229.

25. Ott, S. M., R. F. Kilcoyne, and C. H. Chesnut III. 1987. Ability of four different techniques of measuring bone mass to diagnose vertebral fractures in postmenopausal women. J. Bone Miner. Res. 2:201210 .

26. Nilas, L., J. Podenphant, B. J. Riis, A. Gotfredsen, and C. Christiansen. 1987. Usefulness of regional bone mass measurements in patients with osteoporosis fractures of the spine and distal forearm. $J$. Nucl. Med. 28:960-965.

27. Podenphant, J., V. A. H. Nielsen, B. J. Riis, A. Gotfredsen, and C. Christiansen. 1987. Bone mass, bone structure and vertebral fractures in osteoporotic patients. Bone. 8:127-130.

28. Mazess, R. B. 1988. Diagnostic sensitivity of bone densitometry (letter). J. Bone Miner. Res. 3:121-122. 